\title{
Rapid endovascular treatment for stroke
}

\section{Faizan Khan BSc, Satya Patro MD, Daniela lancu MD MSc}

\section{Competing interests: \\ None declared.}

This article has been peer reviewed.

The authors have obtained patient consent.

Affiliation: Division of

Neuroradiology,

Department of Radiology,

The Ottawa Hospital,

Ottawa, Ont.

Correspondence to:

Daniela Iancu,

diancu@toh.on.ca

CMAJ 2016. DOI:10.1503 /cmaj.150633
Interventional

A n 87-year-old woman presented to the emergency department three hours after acute onset of left hemiplegia, profound neglect and dysarthria (severe stroke; National Institutes of Health Stroke Scale [NIHSS] $=18$ ). Noncontrast computed tomography (CT) of the head showed early ischemic changes in the right putamen and insula, along with a hyperdense right middle cerebral artery (MCA) sign (i.e., hyperdense clot in the right M1 segment of the MCA). Computed tomography angiography showed occlusion of the right internal carotid artery terminus, with good collateral circulation in the right MCA territory (for CT images, see Appendix 1, available at www.cmaj.ca/lookup/ suppl/doi:10.1503/cmaj.150633/-/DC1). Intravenous tissue plasminogen activator was administered 30 minutes after arrival.

Given her clinical status and favourable imaging results ("good-scan-occlusion paradigm,"1 i.e., moderate to severe stroke, large-vessel occlusion, minimal early ischemic changes and good collateral circulation), the patient was considered a suitable candidate for intra-arterial thrombectomy. She was immediately transferred to the angiography suite, where catheter angiog-

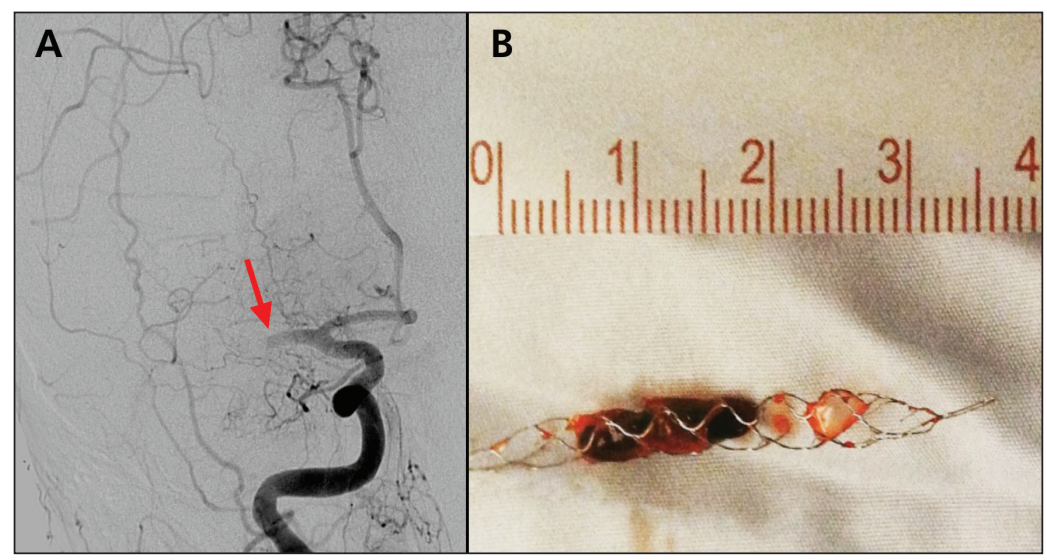

Figure 1: (A) Pre-thrombectomy angiogram of the right internal carotid artery of an 87-year-old woman with severe stroke, showing complete occlusion of the mid M1 segment of the right middle cerebral artery (red arrow). (B) Thrombus (length about $1.4 \mathrm{~cm}$ ) extracted from the right middle cerebral artery with a stent-retriever thrombectomy device. raphy of the neck and head indicated occlusion of the right mid-to-distal M1 segment of the right MCA (Figure 1A).

We performed mechanical thrombectomy by positioning a stent-retriever thrombectomy device in the distal M1 segment of the right MCA, alongside the target thrombus. The stent was deployed for five minutes before being gradually retrieved, along with the captured blood clot (Figure 1B). Postprocedural angiography showed complete recanalization of the right MCA (Appendix 2, available at www.cmaj.ca/lookup/ suppl/doi:10.1503/cmaj.150633/-/DC1), achieved about 17 minutes after inguinal puncture. Head CT performed 24 hours after reperfusion showed no major complications. The patient's left hemiplegia diminished markedly, and she was discharged on day 12 , with minimal residual weakness and dysarthria (NIHSS $=6$ ).

Numerous studies have emphasized the effectiveness of timely intra-arterial thrombectomy with stent-retriever devices, combined with intravenous tissue plasminogen activator, in markedly improving functional outcome ${ }^{2}$ and reducing mortality ${ }^{3}$ among patients with stroke. These landmark randomized clinical trials provide evidence for a more rapid rate of recanalization of large-vessel occlusion relative to tissue plasminogen activator alone, sometimes within minutes. ${ }^{4}$ Researchers consider the remarkable potency of this procedure as a major breakthrough in the disease, offering a safe and efficient treatment for severe stroke. ${ }^{2}$

\section{References}

1. Bal S, Bhatia R, Shoba N, et al. Stroke-on-awakening: safety of CT-CTA based selection for reperfusion therapy. Can J Neurol Sci 2014;41:182-6.

2. Berkhemer OA, Fransen PS, Beumer D, et al. A randomized trial of intraarterial treatment for acute ischemic stroke. $N$ Engl $J$ Med 2015;372:11-20.

3. Demchuk AM, Goyal M, Menon BK, et al. Endovascular treatment for Small Core and Anterior circulation Proximal occlusion with Emphasis on minimizing CT to recanalization times (ESCAPE) trial: methodology. Int J Stroke 2015;10: 429-38.

4. Campbell BC, Mitchell PJ, Kleinig TJ, et al. Endovascular therapy for ischemic stroke with perfusion-imaging selection. $N$ Engl J Med 2015;372:1009-18. 